Julia Kneisel

\title{
Still und heimlich - der neueste Referentenentwurf des BMJV zur Korruptionsbekämpfung, seine Kernpunkte und Konsequenzen
}

Am 13.06.2014 veröffentlichte das Bundesministerium der Justiz und für Verbraucherschutz einen umfangreichen Referentenentwurf zur Korruptionsbekämpfung. ${ }^{1}$ Eine Pressemitteilung des BMJV oder Medienberichte sind zu diesem Vorhaben bis zum jetzigen Zeitpunkt nicht zu finden, obwohl der Entwurf eine umfassende Einbeziehung europäischer und ausländischer Amtsträger in das Strafgesetzbuch sowie eine Reform des $\$ 299$ StGB (Bestechlichkeit und Bestechung im geschäftlichen Verkehr) vorsieht. Die Amtsträger und Richter anderer Staaten sollen gemäß Entwurf durch eine neue "Gleichstellungsvorschrift“2 ( $\$ 335$ a StGB) vom Anwendungsbereich der $\mathbb{S} 3331 \mathrm{ff}$. StGB erfasst werden. Ebenso werden auch europäische Amtsträger inländischen Amtsträgern gleichgestellt. Insoweit folgt der Entwurf europarechtlichen Vorgaben. Dieses Reformanliegen ist deshalb gerechtfertigt, weil nicht nur das Vertrauen in die Nichtkäuflichkeit inländischer, sondern auch europäischer Amtsträger schützenswert ist. Ob die Regelungen freilich im Einzelnen gelungen und systematisch sorgfältig sind, muss einer separaten Analyse vorbehalten bleiben. ${ }^{3}$ Bereits auf den ersten Blick ergeben sich sprachliche Ungenauigkeiten ${ }^{4}$ und es entsteht unklares Strafrecht durch sich überschneidende Regelungen ${ }^{5}$ nach geltendem und künftigem Recht.

1 Entwurf eines Gesetzes zur Bekämpfung der Korruption; abrufbar unter http://www.bmjv.de /SharedDocs/Downloads/DE/pdff/Gesetze/RefE_KorrBekG.pdf?_blob=publicationFile (Stand 07.07.2014).

2 Nach Referentenentwurf, 18.

3 Ferner wird der Straftatbestand der Geldwäsche durch die Ausdehnung des Vortatenkatalogs $\left(\mathbb{S} \int 299,335 \mathrm{a}\right.$ StGB) erweitert. Bezüglich der weiteren Änderungen, die nicht allein die Korruptionsdelikte, bzw. Delikte, die im Zusammenhang mit Korruption stehen, betreffen (u.a. \S 202c Abs. 1, 78b Abs. 6 StGB), wird im Referentenentwurf auf Artikel 1 Nrn. 4 und 5 verwiesen.

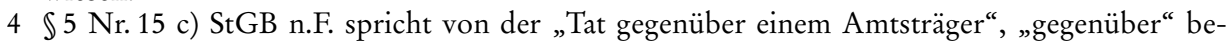
schreibt aber sinngemäß „zum Nachteil von“. Dies ist bei den opferlosen Korruptionsdelikten nicht sachgemäß, die Tat wird auch nicht zum Nachteil des Amtsträgers begangen. Zweckdienlicher wäre die Verwendung der Begriffe „Vorteilsnehmer/Vorteilsgeber“.

5 So wird bereits $\mathbb{5}$ Nr. 15 b) StGB a.F. durch die Reform des Amtsträgerbegriffs in den $\int \mathbb{S} 331 \mathrm{ff}$. StGB und dem Territorialitätsprinzip erfasst. 
Neben den genannten Reformen kommt es zu Neukriminalisierungen im Bereich des $\ 299$ StGB, dessen Reform im vorliegenden Beitrag im Mittelpunkt stehen soll.

\section{A. Nene Probleme aus alten Schubladen: Die Reform des $\int 299$ StGB}

Der Kernpunkt des Entwurfs - die Reform des $\$ 299$ StGB - baut auf Vorhaben vergangener Legislaturen auf. ${ }^{6}$ Die Reform des $\$ 299$ StGB setzt den Trend zur Punitivität im Bereich des Wirtschaftsstrafrechts im Allgemeinen und der Korruptionsdelikte im Besonderen in exemplarischer Weise fort und wird $\mathrm{zu}$ weiteren Rechtsunsicherheiten in der Praxis führen. ${ }^{7}$ Im Einzelnen:

\section{Die Entwicklung und Reformdiskussion der Bestechungsdelikte seit 1997}

Das rechtspolitische Ressort der Korruptionsbekämpfung hat sich seit $1997 \mathrm{zu}$ einer „Gretchenfrage“ entwickelt. In der Außenkommunikation werden die Nichtakzeptanz und der Bekämpfungswille gegenüber jedem dem Anschein nach korrupten Verhalten in Wirtschaft und Gesellschaft von allen Akteuren ${ }^{8}$ beschworen. Im inner circle, insbesondere der Normadressaten (Wirtschaftsunternehmen, Berufsverbände) ${ }^{9}$ oder der

6 Zuletzt und wortlautgleich „Der Entwurf eines ... Strafrechtsänderungsgesetzes“, BR-Drucks. 548/07 v. 10.08.2007.

7 So auch schon Schneider NK 2012, $30 \mathrm{ff}$.

8 Aussagen aus den Wahlprogrammen der politischen Parteien zum Bundestagswahlkampf 2013: „CDU und CSU treten dafür ein, Korruption, Spiel- und Wettmanipulation entschieden zu begegnen.“ (45), „Wir setzen uns ein für eine verfassungskonforme Ratifizierung der UNKonvention gegen Korruption.“ (FDP, 88), „Die Bekämpfung von Korruption braucht eine klare Gesetzgebung mit konsequenter Durchsetzung,(...)“ (Bündnis 90/Die Grünen, 126), „Korruption und Bestechung, (...) Intransparenz und Parteiensponsoring dürfen nicht Mittel der Einflussnahme sein.“ (Die Linke, 74), „Die Piratenpartei setzt sich auf Bundes-, Länderund Kommunalebene für gesetzliche Grundlagen zur systematischen Korruptionsprävention und -verfolgung ein.“ (160), „Korruption und Bestechlichkeit müssen scharf geahndet werden." (SPD, 99); zu den führenden Interessengruppen zählen u.a. Transparency International e.V., das Deutsche Netzwerk Wirtschaftsethik, das Deutsche Forum Kriminalprävention sowie LobbyControl; Stellungnahme des DGB zum „Entwurf eines Gesetzes zur Einrichtung eines Registers über unzuverlässige Unternehmen (Korruptionsregister-Gesetz) Drucksache 17/11415“ v. 22.02.2013 (http://www.dgb.de/themen/++co++bbf7f530-7cde-11e2-aca0-00188 b4dc422); siehe auch Leitartikel in der deutschen Medienlandschaft: „Leitplanken gegen Lobbyisten“ (TAZ 12.05.2013, http://www.taz.de/!115754/), „Deutschland will UN-Konvention gegen Korruption ratifizieren“ (DIE ZEIT, 28.05.2014, http://www.zeit.de/politik/deutschlan d/2014-05/un-bundeskabinett-korruption-konvention) u.a.

9 "BPI nimmt Stellung zur Korruptionsbekämpfung im Gesundheitswesen" vom 15.05.2013 (http://www.bpi.de/home/nachrichten/nachrichten/bpi-nimmt-stellung-zur-korruptionsbeka empfung-im-gesundheitswesen/), „vfa - Transparenz contra Korruption“ v. 18.12.2013 (http:// www.vfa.de/de/verband-mitglieder/transparenzkodex-der-pharmaindustrie/transparenz-cont ra-korruption.html); vgl. auch Ausschussdrucks. 17(14)0424(7) v. 12.05.2013 (Stellungnahme des Verbraucherzentrale Bundesverbandes: "Keine halben Sachen: Gesetzentwurf zur Prävention überarbeiten! "); Ausschussdrucks. 17(14)0424(9) v. 14.05.2013 (Stellungnahme des GKVSpitzenverbandes zum Gesetzesentwurf der Fraktionen der CDU/CSU und FDP zum Ent-

NK 26. Jg. 4/2014 
Wissenschaft, ${ }^{10}$ geht man jedoch meist bedachter mit Kriminalisierungsvorhaben und dogmatischen Neubauten im Strafrecht um. Die politischen Akteure, Parteien in ihren Programmen (s.o.) wie auch Ministerien in ihren Vorhaben, ${ }^{11}$ sehen sich ihrerseits in einem Spannungsfeld von actio und reactio der lobbyhaften Interessengruppen. Resultat dieses Spannungsverhältnisses ist in der Regel ein Kompromiss, wie man etwa an dem Korruptionsbekämpfungsgesetz von 1997 sehen kann. ${ }^{12}$ Der Trend zur Verschärfung vollzieht sich nach diesem Muster in Stufen. Dem Gesetz zur Bekämpfung einer bestimmten Erscheinungsform der Kriminalität folgt einige Jahre später das Gesetz zur Verbesserung der Bekämpfung. ${ }^{13}$ Diesem Algorithmus gehorcht auch die Korruptionsbekämpfung:

Mit der Einführung des $\$ 299$ StGB im Jahr 1997 durch Art. 1 Abs. 3 KorrBekG ${ }^{14}$ hat der Gesetzgeber die Korruption vom Abstellgleis des $\$ 12$ UWG a.F. in das Kernstrafrecht transferiert und damit seine Bedeutung akzentuiert. Der $\$ 12$ UWG a.F. war in der Praxis nahezu bedeutungslos: In der Polizeilichen Kriminalstatistik (PKS) zum Berichtsjahr 1997 wurden 198 Fälle dem $\$ 12$ UWG a.F. zugeordnet. ${ }^{15}$ Dabei handelte es sich lediglich um 1,95\% aller erfassten Fälle der Amts- und Korruptionsdelikte. ${ }^{16}$ Mit der Einführung des $\$ 299$ StGB und seiner Erfassung ab der PKS zum Berichtsjahr $2000^{17}$ stiegen die Zahlen von $124^{18}$ (im Jahr 2000) auf $324^{19}$ (im Jahr 2002) und bis zum Berichtsjahr 2010 auf $761^{20}$ erfasste Fälle an. Mit dem neuen Tatbestand sollte das Bewusstsein dafür geschärft werden, „dass es sich auch bei der Korruption im geschäftlichen Betrieb um eine Kriminalitätsform handelt, die nicht nur die Wirtschaft selbst betrifft, sondern Ausdruck eines allgemein sozialethisch missbilligten Verhaltens

wurf eines Gesetzes zur Förderung der Prävention); Ausschussdrucksache 17(14)0424(8) v. 14.05.2013 (BVMed-Stellungnahme anlässlich der öffentlichen Anhörung am 15.05.2013 zum Entwurf eines Gesetzes zur Förderung der Prävention); s. auch BDI „Stellungnahme zum Entwurf eines Gesetzes zur Einrichtung eines Registers über unzuverlässige Unternehmen (Korruptionsregister-Gesetz)“ v. 21.02.2013 (http://www.bdi.eu/download_content/Rec htUndOeffentlichesAuftragswesen/BDI-STN_KorruptionsregisterG_21.02.13.pdf).

10 Kritisch zur übermäßigen Kriminalisierung: Wienke/Janke/Kramer MedR 2011, 81ff.; Kempf/Lü̈erssen/Volk 2009; Geis GesR 2011, 641 ff.; Steinhilper MedR 2010, 499 ff.; Schneider/Ebermann HRRS 2013, 219 ff.; Schneider StV 2010, 365 ff.; ders. HRRS 2010, 241 ff.

11 Zum Entwurf des Gesetzes zur Förderung der Prävention

(BT-Drucks. 17/13080, 17/14184, 17/14205) wurde der Vermittlungsausschuss angerufen (http://dip21.bundestag.de/dip21/btd/17/147/1714791.pdf).

12 Zur Diskussion standen die Gesetzesentwürfe von SPD-Fraktion (BT-Drucks. 13/8083 v. 26.06.1997) und der CDU/CSU- und FDP-Fraktion (BT-Drucks. 13/8082 v. 26.06.1997).

13 So z.B. bei Organisierter Kriminalität oder Terrorismus.

14 Gesetz zur Bekämpfung der Korruption 1997, vom 13.08.1997, BGBl. I 2038, in Kraft seit dem 20.08.1997.

15 Polizeiliche Kriminalstatistik (BKA) Berichtsjahr 1997, 205 ff.

16 Im Jahr 1997 wurden insgesamt 10.136 Fälle den Amts- und Korruptionsdelikten zugeordnet.

17 Dieses Berichtsjahr wird allgemein als Ausgangsstatistik für die Erfassung des $\$ 299$ StGB gesehen.

18 PKS 2000, 215.

19 PKS 2002, 273.

20 PKS 2010, 221. 
ist“. ${ }^{21}$ In einem ersten Schritt wurden im Gegensatz zum $\ 12$ UWG a.F. Drittvorteile in die Strafbarkeit mit aufgenommen. ${ }^{22}$

Im Jahr $2002^{23}$ erweiterte der Gesetzgeber in einem zweiten Schritt den Anwendungsbereich von Abs. 1 und 2 des $\$ 299$ StGB im Zuge einer nunmehr international geführten Diskussion um Korruptionsbekämpfung auf „Handlungen im ausländischen Wettbewerb“ (Abs. 3). Die nächste Etappe der Diskussion bezog sich auf eine Auslegungsfrage des $\$ 299$ de lege lata. Fokussiert wurden die Berufsgruppe der niedergelassenen Vertragsärzte, bei der unklar war, ob diese im Sinne von Beauftragten der GKV in den Anwendungsbereich des $\$ 299$ StGB fallen. ${ }^{24}$ Einen vorläufigen Abschluss der Diskussion nach geltendem Recht schaffte der Große Senat des BGH durch seinen Beschluss am 29.03.2012 ${ }^{25}$ und konstatierte, dass ein niedergelassener Vertragsarzt weder Beauftragter der gesetzlichen Krankenkassen iSd $\ 299$ StGB noch Amtsträger iSd $\ 11$ Abs. 1 Nr. 2c StGB ist. Diese Entscheidung rief massive Kritik in der Öffentlichkeit hervor. ${ }^{26}$ Damit war es die Obliegenheit des Gesetzgebers, durch künftiges Recht die Voraussetzungen für eine Strafbarkeit von niedergelassenen Vertragsärzten zu schaffen. $^{27}$ In der vergangenen Legislaturperiode ${ }^{28}$ standen sich der Entwurf der Regierungskoalition $^{29} \mathrm{zu}$ entsprechenden Regelungen $\$ \int 307$ c, 70 Abs. 3 im SGB V, und der Entwurf des Bundesrates ${ }^{30}$ zur Einführung eines $\ 299$ a StGB gegenüber. Sie fielen der Diskontinuität anheim. Mit Beginn der 18. Legislaturperiode ${ }^{31}$ brachten die regierungsbeteiligten Parteien in ihrem Koalitionsprogramm das Thema Korruptionsprävention im Gesundheitswesen erneut auf das Polittableau. ${ }^{32}$ Parallel zu diesem Vorhaben soll jetzt $\$ 299$ StGB reformiert und erweitert werden.

21 BT-Drucks. 13/5584, S. 15; BT-Drucks. 553/96, S. 32; kritisch zur Übernahme in das StGB etwa Dölling 1996 Gutachten, C 85 sowie König JR 1997, 397, 401; ebenso entsprechende Einwände des Bundesrates (BT-Drucks. 13/6424, 7).

22 Weiterführend dazu Albus 2007, 22.

23 Mit dem Gesetz zur Ausführung (...) der Gemeinsamen Maßnahme betreffend die Bestechung im privaten Sektor vom 22.12.1998 (...) vom 22.08.2002 (BGBl. I, 3387); in Kraft seit dem 30.08.2002.

24 Pragal NStZ 2005, 133, $134 \mathrm{ff}$.

25 BGH, Beschl. vom 29.03.2012-GSSt 2/11.

26 Geiger CCZ 2012, $172 \mathrm{ff}$.

27 Dannecker ZRP 2013, 39ff.

28 Gemeint ist hier die durch CDU/CSU und FDP geführte 17. Legislaturperiode.

29 Entwurf des Gesetzes zur Förderung der Prävention, BT-Drucks. 17/13080, 17/14184, 17/14205. Der Entwurf wurde im Rahmen des als „Omnibusgesetz“ konzipierten Präventionsgesetzes als ein Verbot der Bestechlichkeit/Bestechung von „Leistungserbringen“ eingebracht.

30 Entwurf eines ... Strafrechtsänderungsgesetzes zur Bekämpfung der Korruption im Gesundheitswesen (... StrÄndG), BR-Drucks. 451/13.

31 Gemeint ist hier die CDU/CSU und SPD geführte 18. Legislaturperiode, die am 22.10.2013 begann.

32 „Wir werden einen neuen Straftatbestand der Bestechlichkeit und Bestechung im Gesundheitswesen im Strafgesetzbuch schaffen." (Koalitionsvertrag, 77).

NK 26. Jg. 4/2014 


\section{Vorgesehene Änderungen im Entwurf des $\$ 299 \mathrm{StGB}$}

Um die Änderungen des $\$ 299$ StGB laut dem Referentenentwurf zu veranschaulichen, werden die bisherige Fassung und der Neuentwurf synoptisch gegenüber gestellt:

\begin{tabular}{|l|l|}
\hline \multicolumn{1}{|c|}{ \$299 StGB - bisherige Fassung } & \multicolumn{1}{|c|}{ \$299 StGB - neue Fassung } \\
\hline $\begin{array}{l}\text { (1) Wer als Angestellter oder Beauftragter eines } \\
\text { geschäftlichen Betriebes im geschäftlichen Ver- } \\
\text { kehr einen Vorteil für sich oder einen Dritten als }\end{array}$ & $\begin{array}{l}\text { (1) Wer im geschäftlichen Verkehr als Angestell- } \\
\text { ter oder Beauftragter eines Unternehmens einen } \\
\text { Vorteil für sich oder einen Dritten als Gegenleis- } \\
\text { tung dafür fordert, sich versprechen lässt oder } \\
\text { Gegenleistung dafür fordert, sich versprechen } \\
\text { läßt oder annimmt, daß er einen anderen bei dem } \\
\text { Bezug von Waren oder gewerblichen Leistun- } \\
\text { gen im Wettbewerb in unlauterer Weise bevor- } \\
\text { oder Dienstleistungen dem Bezug von Waren } \\
\text { zuge, wird mit Freihenen anderen im inländischen oder auslän- } \\
\text { oder mit Geldstrafe bestraft. } \\
\text { dischen Wu drei Jahren } \\
\text { vorzuge oder } \\
\text { 2. seine Pflichten gegenüber dem Unterneh- } \\
\text { men verletze, } \\
\text { wird mit Freiheitsstrafe bis zu drei Jahren oder } \\
\text { mit Geldstrafe bestraft. }\end{array}$ \\
\hline \multicolumn{2}{|c|}{ Abs. 2 spiegelbildlich zu Abs. 1 für den Vorteilsgeber. } \\
\hline (3) Die Abs. 1 und 2 gelten auch für Handlungen & Gestrichen \\
im ausländischen Wettbewerb. &
\end{tabular}

Zuerst handelt es sich um einige redaktionelle Umstellungen, wie die Vorziehung „im geschäftlichen Verkehr" sowie die Streichung des Abs. 3 durch seine Einsetzung in Nr. 1 der Abs. 1 und 2, die jedoch offensichtlich keine inhaltlichen Auswirkungen haben. Nummer 1 der Abs. 1 und 2 entspricht unverändert der bisherigen Rechtslage.

Näher zu betrachten ist allerdings, dass nunmehr statt der Zugehörigkeit zu einem „geschäftlichen Betrieb“ die zu einem „Unternehmen“ gelten soll. Der Referentenentwurf begründet dies mit der redaktionellen Änderung im Gesetz gegen den unlauteren Wettbewerb, ${ }^{33}$ durch die in diesem Gesetz der Begriff des geschäftlichen Betriebes durch den Begriff des Unternehmens ersetzt wurde. Dasselbe betreffe auch die Änderung von "Waren und gewerblichen Leistungen" in "Waren und Dienstleistungen“. ${ }^{34}$ Somit ist Ziel des Referentenentwurfs, den Sprachgebrauch gesetzesübergreifend zu harmonisieren.

Eine zusätzliche Neueinführung stellt Nummer 2 der Abs. 1 und 2 dar. Die europarechtlichen Regelungen ${ }^{35}$ verlangten, so die Entwurfsbegründung, eine Strafbarkeit für den Fall, dass der Vorteilsnehmer als Gegenleistung für die Vorteilszuwendung eine Handlung vornehmen oder unterlassen soll, die seine Pflichten gegenüber dem Unternehmen verletzt. Diese Pflichtverletzung, gemäß Wortlaut des Entwurfs, führt zu einer

33 Vom 03.07.2004 (BGBl. I 2004, 1414).

34 Referentenentwurf, 22.

35 Laut Art. 2 EU-Rahmenbeschluss (zur Bekämpfung der Bestechung im privaten Sektor) vom 22.07.2003 (Abl. L 192 vom 31.07.2003, 54) sowie Art. 7 und 8 des Europarat-Übereinkommens (ETS Nummer 173), so der Referentenentwurf, 21. Eine entsprechende unverbindliche Vorgabe sieht auch Art. 21 des VN-Übereinkommens vom 31.10.2013 gegen Korruption vor. 
Strafbarkeit, ohne dass es auf den Eintritt eines Vermögensschadens bei dem Unternehmen ankommt. Jeweilige redaktionelle Angleichungen finden sich in Bezug auf $\$ 299 \mathrm{StGB}$ in den $\$ \$ 301$ und $302 \mathrm{StGB} .^{36}$

\section{Auslegungsprobleme und Konsequenzen}

Die oben dargestellten Änderungen führen zu mehreren Fragestellungen. Einfache Antworten, wie der Referentenentwurf sie suggeriert, lassen sich darauf nicht ohne weiteres finden. Im Folgenden sollen vier Probleme aufgezeigt und diskutiert werden:

\section{Problem: „Dienstleistungen“ im $\ 299$ StGB}

\$299 StGB a.F. erfasst die Bevorzugung beim Bezug von Waren oder gewerblichen Leistungen. Im Sinne des Schutzzweckes der Norm ist damit „das gesamte, auf die Erlangung oder den Absatz von Waren oder Leistungen, gerichtete Geschäft “ 37 gemeint. Fielen darunter nur gewerbliche Leistungen im engeren Sinne, würde der freie Wettbewerb nicht mehr umfassend geschützt und die Vorschrift wäre in der Praxis von geringer Bedeutung. ${ }^{38}$

Wie bereits oben dargestellt, ist laut Referentenentwurf mit Verweis auf die Änderung im UWG eine Änderung von „gewerbliche Leistungen“ in „Dienstleistungen“ im \$299 StGB vorgesehen. Nach \2 UWG sind Dienstleistungen „alle geldwerten unkörperlichen Leistungen, die im geschäftlichen Verkehr erbracht werden können“. Hierunter versteht man die Leistungen der gewerblichen Wirtschaft, aber auch die der freien Berufe, so die Leistungen der Ärzte, Rechtsanwälte, Handelsvertreter, der Vermietungs- und Wartungsdienste, der Finanzierungs- und Versicherungsleistungen usw. ${ }^{39}$ Insofern führt die Wortlautänderung daher nicht zu einer inhaltlichen Veränderung des \299 StGB.

2. Problem: Der neu eingeführte Begriff des „Unternehmens“ im $\ 299 \mathrm{StGB}$

Bislang musste der Täter Angestellter oder Beauftragter eines geschäftlichen Betriebes sein. Der geschäftliche Betrieb „umfasst jede auf Dauer bestimmte, regelmäßige Teilnahme am Wirtschaftsleben mittels Leistungsaustauschs. “40 Nicht als taugliche Täter angesehen wurden Angestellte oder Beauftragte von Privatpersonen. ${ }^{41}$

36 Siehe Referentenentwurf, Nrn. 12 und 13, 5f.

37 MüKo-Krick 2014 StGB, $\mathbb{\$} 299$, Rn. 26.

38 So auch BGH v. 2.5.1957 - 4 StR 119-120/56; BGHSt 10, 269, 270; Kindhäuser/Neumann/ Paeffgen-Dannecker 2013 StGB \299, Rn 55; Dann wistra 2011, 127, 129; MüKo-Krick 2014 StGB, \299, Rn. 26.

39 Obly/Sosnitza 2014 UWG, $\$ 2$, Rn. 48.

40 BGHSt 2, 396, 403; 10, 359, 366.

41 Kindhäuser/Neumann/Paeffgen-Dannecker 2013 StGB, \299, Rn. 24.

NK 26. Jg. 4/2014 
Als Begründung für die Ersetzung des Wortes „geschäftlicher Betrieb“ durch „Unternehmen" verweist der Referentenentwurf ebenfalls auf das Gesetz gegen den unlauteren Wettbewerb. ${ }^{42}$ Das UWG verwendet jedoch nicht den Begriff des „Unternehmens“, sondern spricht stattdessen vom „Unternehmer“. Eine Änderung im Regelungsinhalt des $\$ 299$ StGB, hin zur Erfassung von Privatpersonen, kann damit aber im Ergebnis gleichwohl nicht gewollt sein.

Das UWG verweist in $\$ 2$ Abs. 2 auf den Begriff des Unternehmers in $\$ 14$ BGB. Gemäß $\$ 14$ BGB ist der Unternehmer „eine natürliche oder juristische Person oder eine rechtsfähige Personengesellschaft, ${ }^{43}$ die bei Abschluss eines Rechtsgeschäfts in Ausübung ihrer gewerblichen oder selbständigen beruflichen Tätigkeit handelt" “. ${ }^{44}$

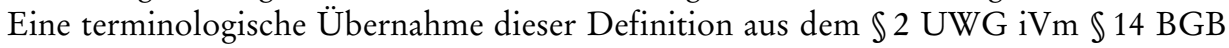
in $\$ 299$ StGB kann aber nicht ohne Wertungswidersprüche und ohne Verstoß gegen die Wortlautgrenze ${ }^{45}$ erfolgen. ${ }^{46}$

Denn nach Wortlaut wird das Unternehmen im Duden als ein „aus mehreren Werken, Filialen o. Ä. bestehender Betrieb (im Hinblick auf seine wirtschaftliche Einheit) “47 bezeichnet. Damit sind Privatpersonen offensichtlich nicht gemeint. Sollen Einzelpersonen erfasst werden, wird dies in der Sprache der Lebenswelt ${ }^{48}$ regelmäßig durch die Verwendung von sich aus zusammengesetzten Substantiven speisenden Neologismen gekennzeichnet, z.B. der Einmannbetrieb. ${ }^{49}$

Alleine diese Interpretation harmonisiert auch mit der Auslegung des $₫ 30$ OWiG. Die Unternehmensgeldbuß $\mathrm{e}^{50}$ wird nicht einzelnen Unternehmern, sondern der juristischen Person auferlegt. ${ }^{51}$

Es muss nach diesen Überlegungen somit davon ausgegangen werden, dass die Änderung vom „geschäftlichen Betrieb“ zum „Unternehmen“ nicht von dem gesetzgeberischen Willen getragen ist, die Strafbarkeit auch auf Angestellte oder Beauftragte von Privatpersonen auszudehnen. Es bleibt jedoch der Hinweis, dass der systematische Verweis des Entwurfs auf das UWG aufgrund begrifflicher Ungleichheit der Definitionsgrundlage fehlläuft.

42 Vom 03.07.2004, BGBl. I 1414.

43 Eine rechtsfähige Personengesellschaft ist eine Personengesellschaft, die mit der Fähigkeit ausgestattet ist, Rechte zu erwerben und Verbindlichkeiten einzugehen, $\mathbb{} 14$ Abs. 2 BGB.

44 Nach $\$ 14$ Abs. 1 BGB; auch freiberufliche Tätigkeiten sind nicht ausgeschlossen, so MüKoMicklitz 2012 BGB, $\ 14$, Rn. 5.

45 Larenz/Canaris 1995, 141.

46 A.A. sind diesbezüglich Rönnau/Golombek ZRP 2007, 193ff., hier wird der Wertungswiderspruch nicht gesehen.

47 http://www.duden.de/rechtschreibung/Unternehmen v. 10.07.2014.

48 Nach Schütz/Luckmann 1979.

49 „Geschäft o. Ä., das nur von einer Person betrieben wird“, http://www.duden.de/rechtschre ibung/Einmannbetrieb v. 10.07.2014.

50 Schmitt-Leonardy 2013, $296 \mathrm{ff}$.

51 Auch kann die Geldbuße nicht-rechtsfähigen Vereinen (iSd $\$ 55$ BGB) oder rechtsfähigen Personengesellschaften (iSd $\$ 14$ Abs. 2 BGB) auferlegt werden, nicht aber natürlichen Personen, Achenbach/Ransiek 2012, $7 \mathrm{f}$. 
3. Problem: Die Einführung des „Geschäftsherrenmodells“ in $₫ 299$ Nr. 2 StGB

Künftig soll nach Änderung des $₫ 299$ StGB der Tatbestand um eine Variante Nr. 2 in den beiden Absätzen ergänzt werden, die dem sogen. „Geschäftsherrenmodell“52 entspricht. Bei diesem Modell der Ausrichtung des $\ 299$ StGB steht statt des Kollektivrechtsguts des funktionierenden Leistungswettbewerbs das Individualinteresse des $\mathrm{Ge}$ schäftsherrn an einer loyalen und unbeeinflussten Pflichterfüllung seiner Angestellten im Fokus. ${ }^{53}$ Danach ist der Tatbestand erfüllt, wenn der Angestellte oder Beauftragte eine Pflicht gegenüber dem Unternehmen verletzt. Welche Pflichtverletzungen hiermit gemeint sind, ist offen.

Es entsteht folglich ein der Untreue ähnlicher Tatbestand, der 1. Abgrenzungsfragen $\mathrm{zu} \$ 266 \mathrm{StGB}$ aufwirft, 2. bereits bestehende Auslegungsprobleme der Untreue multipliziert und 3. zu einer problematischen Verschiebung des Rechtsgutes des $\ 299 \mathrm{StGB}$ führt.

a) Fallbezogene Abgrenzungsfragen zwischen $\$ 266$ und $\$ 299$ StGB a. u. n. F.

De lege lata ist zwischen $\$ 299$ a.F. und $\$ 266$ StGB folgendermaßen abzugrenzen:

Bisher erfüllt der Täter $\$ 299$ StGB a.F. z.B. dann, wenn er als Angestellter oder Beauftragter eines geschäftlichen Betriebes durch Bevorzugung im Rahmen der Auftragsvergabe an einen Dritten eine vertragliche Pflicht (z.B. eine Vermögensbetreuungspflicht) verletzt und im Gegenzug einen Vorteil erhält. Ob dadurch ein Vermögensschaden entsteht, ist für $\ 299 \mathrm{StGB}$ a.F. irrelevant.

\266 StGB erfasst demgegenüber alle Fallkonstellationen, in denen der Täter seine Vermögensbetreuungspflicht verletzt und ein Vermögensschaden entsteht, seine Pflichtverletzung aber nicht durch Vorteile motiviert war.

Bereits jetzt gibt es eine Schnittmenge zwischen $\$ 299$ StGB a.F. und $\$ 266$ StGB. Diese liegt vor, wenn der Täter im Rahmen der Auftragsvergabe an einen Dritten seine Vermögensbetreuungspflicht verletzt (z.B. in Form einer Kick-Back-Abrede) und dadurch ein Vermögensschaden bei dem geschäftlichen Betrieb (Unternehmen) entsteht. Diese Fallkonstellation bildet in der Praxis den Regelfall, weil gezahlte Kick-Backs (Rückvergütungen) üblicherweise auf den Preis aufgeschlagen werden. ${ }^{54} \mathrm{Nach}$ Ansicht der Rechtsprechung besteht zwischen $\$ 299$ StGB a.F. und $\$ 266$ StGB regelmäßig55 Idealkonkurrenz, Einzelheiten sind strittig.

Insofern ist die Nummer 2 des $\$ 299$ Abs. 1 und 2 StGB n.F. über die bisher bereits durch das geltende Strafrecht erfassten Fälle hinaus dann einschlägig, wenn der Täter eine Pflichtverletzung begeht, eine Bevorzugung eines Anderen aber nicht vorliegt oder zumindest nicht nachweisbar ist und/oder (noch) kein Vermögensschaden vorliegt.

52 Vogel 2004, 395ff.

53 Rönnau/Golombeck ZRP 2007, $193 \mathrm{f}$.

54 Vgl. z.B. BGH 1 StR 820/81; 1 StR 614/93; 1 StR 274/85.

55 Näher dazu BGH NStZ 2009, 445, 446.

NK 26. Jg. 4/2014 
Beispiel: Angestellter $G$ soll für das Unternehmen W eine nene Büromöbelausstattung erwerben. Nach gründlicher Angebotssichtung entdeckt $G$ den Anbieter Y. G wird von $Y$ zur Produktpräsentation und Auftragsabsprache auf dessen Yacht vor Mallorca eingeladen. Die Annabme solcher Einladungen von potenziellen Auftragnehmern ist nach unternehmensinterner Richtlinie nicht zulässig. G nimmt diese Einladung dennoch an. Schließlich entscheidet sich $G$ in der Auftragsvergabe für den Anbieter $Y$. Y ist auch der günstigste und qualitativ beste Anbieter für das Unternehmen $W$.

G hat sich nach $\$ 299$ Abs. 1 Nr. 2 StGB n.F. strafbar gemacht. Die Einladung auf die Yacht des $Y$ ist ein Vorteil iSd $\$ 299$ StGB. Eine Bevorzugung des Y durch G ist nicht nachweisbar, jedoch hat $G$ gegen die Unternehmensrichtlinie, deren Einhaltung eine arbeitsvertragliche Pflicht ist, verstoßen. Y hat sich nach $\$ 299$ Abs. 2 Nr. 2 StGB n.F. strafbar gemacht. Nach dem geltenden Recht hätte sich G nicht nach $\$ 299$ StGB strafbar gemacht, weil die unlautere Bevorzugung nicht gegeben ist. $\$ 266$ StGB scheitert mangels Vermögensschaden.

Offensichtlich ist, dass der Anwendungsbereich der Nummer 2 arbeitsvertraglichen Ungehorsam, z.B. gegenüber unternehmensinternen Richtlinien zu Compliance, zu einem strafwürdigen und strafbedürftigen Verhalten im Rahmen des staatlichen Sanktionsrechts aufwertet. Es handelt sich bei der Nummer 2 also um eine Neukriminalisierung von Verhalten, dessen Bewertung vorher allein arbeitsrechtlichen Regelungen und dem Sanktionierungsermessen des Unternehmers oblag.

b) Künftige Rechtsanwendungsprobleme, auch im Zusammenhang mit $\ 266 \mathrm{StGB}$

Wie bereits angedeutet, finden sich in der Neueinführung der Pflichtverletzung gegenüber dem Unternehmen eine Vielzahl der Auslegungsprobleme wieder, die bereits im Rahmen des $\$ 266$ StGB heftig diskutiert werden.

Die Pflichtverletzung iSd Nummer 2 ist als offener Rechtsbegriff ausgestaltet und kann daher jede Pflichtverletzung bedeuten. Die Verfasser des Referentenentwurfs sehen darin wohl keine Hinderungsgründe. Jedoch bereitet bekanntlich schon die Konkretisierung der Vermögensbetreuungspflicht (-verletzung) iSd $\$ 266$ StGB Schwierigkeiten und es werden immer wieder Bedenken im Hinblick auf einen Verstoß gegen den Bestimmtheitsgrundsatz geltend gemacht. ${ }^{56} \mathrm{Nach}$ der Rechtsprechung des $\mathrm{BGH},{ }^{57}$ die in der Literatur ${ }^{58}$ nicht unumstritten ist, sollen deshalb nur "gravierende“ Pflichtverletzungen unter den Tatbestand des $\$ 266$ StGB fallen.

Hinsichtlich des $₫ 299$ Nr. 2 n.F. wäre deshalb zu überlegen, ob das genannte einschränkende Kriterium hier ebenfalls hinzuzuziehen ist. Andererseits wertet die Rechtsprechung ${ }^{59}$ im Rahmen einer „Prozeduralisierung“ des Begriffs der Pflichtver-

56 Schönke/Schröder-Perron 2014 StGB, \266, Rn. 11ff.; Saliger JA 2007, 326ff.

57 BGH 47, 150, 197; ebenso Saliger NJW 2010, 3195, 3196; MüKo-Dierlamm 2014 StGB, \$266, Rn. 175; Kiethe NStZ 2005, 531.

58 Z.B. Schünemann NStZ 2005, 473, 475; Fischer 2014 StGB, \266, Rn. 61.

59 BGH 51, 112, 113. 
letzung interne Compliance-Richtlinien strafrechtlich auf, indem unter bestimmten Voraussetzungen aus einem Verstoß gegen die Compliance-Richtlinie auf das Vorliegen einer Pflichtverletzung iSd $\$ 266$ StGB geschlossen wird. Insoweit ist, wie das obige Beispiel der Vertragsverhandlung auf der Yacht im Mittelmeer zeigt, zu befürchten, dass insbesondere bei $\$ 299$ Nr. 2 StGB n.F. jede Verletzung interner Compliance-Vorgaben mit einer Pflichtverletzung iSd Nr. 2 gleichgesetzt wird.

Zudem fehlt bei $\$ 299$ Nr. 2 StGB n.F. im objektiven Tatbestand das Kriterium des Vermögensschadens, das der strafrechtlichen Verantwortlichkeit bei den Fallkonstellationen der Untreue berechenbare Grenzen gibt. Der Vorteilsbegriff, der sich in $\$ 299$ StGB findet, kann eine vergleichbare Grenzziehungsfunktion nicht leisten, weil auch er zu den zwar kernprägnanten, nicht aber randscharfen Rechtsbegriffen gehört und von der Rechtsprechung weit ausgelegt wird. ${ }^{60}$

Ein weiteres Problem besteht in der Umgehung der fehlenden Versuchsstrafbarkeit bei $₫ 266$ StGB durch $₫ 299$ Nr. 2 n.F. Dies hat Auswirkungen für den Täter und den Teilnehmer einer bisher straflosen versuchten Untreue.

Denn verletzt der Täter de lege lata seine Vermögensbetreuungspflicht, ohne dass ein Schaden eintritt, bleibt die Untreue im nicht strafbaren Versuchsstadium stecken. $\mathrm{Da}$ es mithin an einer teilnahmefähigen Haupttat fehlt, gilt dies auch für einen potentiellen Anstifter zur Untreue. Dieses Ergebnis ändert sich durch $\$ 299$ Nr. 2 n.F.

Erklärt sich der Angestellte oder Beauftragte gegenüber dem Vorteilsgeber lediglich bereit, seine Pflichten ${ }^{61}$ gegenüber dem Unternehmen zu verletzten, ist er nach neuer Rechtslage gemäß $\$ 299$ Abs. 1 Nr. 2 StGB strafbar. Auf den Eintritt eines Vermögensschadens kommt es nicht an. Der Vorteilsgeber ist sogar dann strafbar, wenn er den Vorteil lediglich anbietet, ohne dass der Vorteilsnehmer sich zu der Pflichtverletzung iSd Nr. 2 bereit erklärt. Dies entspricht strukturell einer versuchten Anstiftung zur Untreue, die nach geltendem Recht selbstredend straflos ist, nach neuer Rechtslage gemäß $\$ 299$ Abs. 2 Nr. 2 StGB indessen kriminalisiert wird. ${ }^{62}$

c) (Neu-) Ausrichtung des Rechtsguts des $\$ 299$ StGB und fragliche Ausdehnung des Schutzbereiches

Nach herrschender Meinung ${ }^{63}$ schützt $\ 299$ StGB a.F. vorrangig das Interesse der Allgemeinheit am fairen, unbeeinflussten Wettbewerb. Daneben sollen auch potentielle Vermögensinteressen von Mitbewerbern und Geschäftsherren geschützt sein, dies ist

60 Schneider 2008 FS Seebode, $331 \mathrm{ff}$.; Schneider NK 2012, $30 \mathrm{ff}$.

61 Gemeint sind hier Vermögensbetreuungspflichten iSd \$266 StGB.

62 Offen ist auch, wie sich das Einverständnis des Geschäftsherrn in die neuen Tatbestandsvarianten auf die strafrechtlichen Folgen auswirkt.

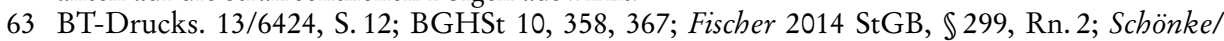
Schröder-Heine/Eisele 2014 StGB, Vor \298, Rn. 5; Lackner/Kübl 2014 StGB, \298, Rn. 1; BGH 49 229, Dölling 1996 Gutachten, C 86.

NK 26. Jg. $4 / 2014$ 
jedoch streitig. ${ }^{64}$ Entgegen der Aussage im Referentenentwurf ${ }^{65}$ kann dieses Rechtsgut nicht ohne weitere dogmatische Probleme als den $\$ 299$ StGB n.F. charakterisierend aufgenommen werden. Die Einführung der Pflichtverletzung gegenüber dem Unternehmen würde den Schutzbereich des $\$ 299$ StGB auf Loyalitätsinteressen des Geschäftsherrn ausdehnen. Hier versucht der Entwurf zwei unterschiedliche Regelungsmodelle im Interesse von Neukriminalisierungswünschen in einer neuen Strafvorschrift zu vermischen - das wettbewerbsstrafrechtliche ${ }^{66}$ und das vermögensstrafrechtliche mit Bezug zu arbeitsrechtlichen Verpflichtungen. ${ }^{67}$ Vor diesem Hintergrund ist auch der systematische Standort des $\$ 299$ StGB n.F. in Abschnitt 26, der mit „Straftaten gegen den Wettbewerb“ überschrieben ist, fraglich.

\section{B. Kritik des Reformvorbabens, insbesondere vor dem Hintergrund des parallelen Vorbabens zur Einfübrung eines $\ 299$ a St $G B$}

Aus den vorherigen Darstellungen ist das Resümee zu ziehen, dass der vorliegende Referentenentwurf zur Reform des $\$ 299$ StGB

1. überflüssig ist, weil die Anwendung des $\$ 299$ StGB n.F. sich auf einen Exklusivbe-

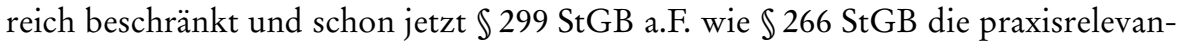
ten Fallkonstellationen in ausreichendem Maße erfassen;

2. schädlich ist, weil die Einführung des konturlosen Merkmals der Pflichtverletzung die bestehenden verfassungsrechtlichen Bedenken, die gegen $\$ 266$ StGB sprechen, potenziert. Auch für die Entwicklung und Implementierung von ComplianceRichtlinien kann sich $\$ 299$ Nr. 2 n.F. als kontraproduktiv erweisen. Wie dargelegt, besteht die Gefahr, dass seitens der Rechtsprechung Verstöße gegen diese internen Verhaltensregularien als strafrechtlich relevante Pflichtverletzung iSd $\$ 299$ Nr. 2 StGB n.F. aufgewertet werden. Diesem Risiko für die eigenen Mitarbeiter können Unternehmen entgehen, wenn sie auf die Implementierung von Compliance-Richtlinien verzichten;

3. übermäßig ist, weil eine zivilrechtliche Nebenpflicht des Angestellten oder Beauftragten gegenüber dem Unternehmen durch eine Strafvorschrift geschützt wird, obwohl weder eine Verzerrung des Wettbewerbs noch ein Vermögensschaden vorliegt. Diese Ausdehnung des Schutzbereiches des $\$ 299$ StGB n.F. ist damit fraglich und durch den Gedanken des Rechtsgüterschutzes kaum zu rechtfertigen.

Zur Verunsicherung trägt gleichzeitig das Projekt der Einführung eines $\$ 299$ a StGB („Bestechlichkeit und Bestechung im Gesundheitswesen“) bei, das in der letzten Legis-

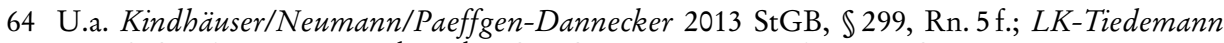
2012 StGB, $\$ 299$, Rn. 1; vgl. auch BGH GRUR 68, 598 zu \12 UWG aF.

65 Referentenentwurf, 21.

66 Mit Bezug zum Rechtsgut des freien Wettbewerbs.

67 Mit Bezug zum Rechtsgut des Schutzes der (Loyalitäts-) Interessen des Geschäftsherrn. 
laturperiode der Diskontinuität anheim fiel ${ }^{68}$ und nun wieder Aufwind erfährt. ${ }^{69}$ Anstelle einer Rechtszersplitterung durch parallele Reformprojekte und die Einführung selektiven Strafrechts $(\$ 299 \text { a StGB })^{70}$ wäre zu überlegen, das Regelungsanliegen des $\$ 299$ a StGB im Rahmen einer umfassenden Reform des $\$ 299$ StGB mit zu berücksichtigen.

\section{Konsequenzen für eine zielfübrende Reform des $\ 299$ St $G B$}

Bisherige Entwürfe zur Reform des $\ 299 \mathrm{StGB}$, die nicht selektiv einzelne Berufsgruppen benachteiligen, fanden freilich keine politische Mehrheit. $\mathrm{Zu}$ nennen ist hier vorrangig der Formulierungsvorschlag von Erb. ${ }^{71}$ Sein Entwurf erfasst auch die Konstellationen der Bestechlichkeit und Bestechung von Selbständigen, die nicht als Beauftragte eines geschäftlichen Betriebes (Unternehmen) agieren und bleibt dem Rechtsgut des freien Wettbewerbs treu. Freiberuflich tätige Ärzte wären somit erfasst, ohne dass ein $\$ 299$ a StGB erforderlich wäre.

Doch die Auseinandersetzung mit dem Strafwürdigem und Strafbedürftigem des Wirtschaftsstrafrechts müsste bereits im vorgesetzgeberischen Stadium stattfinden. Das Gesetzesvorhaben der GroKo folgt einem Übermaß an Forderungen aus Politik, ${ }^{72}$ Wissenschaft ${ }^{73}$ und Praxis, ${ }^{74}$ deren Kriminalisierungswünsche Modewellen unterworfen sind und sich heute u.a. ${ }^{75}$ auf die Zielgruppe der Wirtschaftsakteure beziehen. Diese rechtspolitische Entwicklung setzt sich, wie Scheerer ${ }^{76}$ in seiner Publikation zum atypischen Moralunternehmer schrieb, seit den 80er Jahren fort: „Es erscheint zuneh-

68 Siehe dazu umfassend Schneider HRRS 2013, S. 473ff. Der Regelungsentwurf der Regierungskoalition sah eine ebenfalls nicht unproblematische Implementierung von $\$ \$ 307 \mathrm{c}, 70$ Abs. 3 SGB V vor (BT-Drucks. 17/13080 v. 16.04.13 Gesetzesentwurf der Fraktionen der CDU/CSU und FDP).

69 Pressemitteilung des Petitionsausschusses des Deutschen Bundestages vom 02.07.2014 (http:/ /bundestag.de/presse/hib/2014_07/-/285962): Petition zur Einführung einer gesetzlichen Regelung im StGB zur Strafbarkeit der Korruption im Gesundheitswesen soll zur Erinnerung an das Vorhaben an das Bundesministerium für Gesundheit sowie das BMJV und an die Fraktionen weitergeleitet werden.

70 BR-Drucksache $451 / 13$ v. 30.05.2013.

71 Erb 2011, 111; ferner Pragal, ZIS 2006, 63ff.

72 Allen voran Transparency International: "[...] Missständen, die allem Anschein nach gravierende finanzielle Belastungen des Gesundheitswesens zur Folge haben, mit Mitteln des Strafrechtes effektiv entgegenzutreten." (aus der Pressemitteilung vom 03.01.2013, http://www.tr ansparency.de/2013-01-03-Kassenaerzte-sankti.2210.0.html?\&contUid=4857).

73 Braun MedR 2013, 277 ff., 284; Dannecker ZRP 2013, 37 ff.; bereits Dieners/Taschke PharmR 2000, 309 ff., 319; ähnlich Kölbel NStZ 2011, 195 ff.

74 Fischer 2014 StGB, $\mathbb{2} 299$, Rn. 10e zur Entscheidung des Großen Senats: "[...] Der GrSen hat die insoweit bestehenden "Missstände" gesehen und an den Gesetzgeber appelliert, bestehende Lücken zu schließen (GSSt 2/11, Rn. 46). Diese Lücken sind rechtspolitisch nicht legitimierbar. [...]“.

75 Zur Entwicklung des Sexualstrafrechts nach Edathy siehe Frommel ZRP 2014, Heft 6, im Druck.

76 Scheerer KrimJ 1986, 1. Beiheft, $133 \mathrm{ff}$.

NK 26. Jg. $4 / 2014$ 
mend unproblematisch, dass der Staat die Verbreitung des „richtigen“ Gesellschaftsbildes und der „richtigen“ Sozialmoral mit der Polizei gewährleisten soll“".77

\section{Literatur:}

Achenbach/Ransiek (2012) Handbuch Wirtschaftsstrafrecht

Albus (2007) Die Zusammenarbeit zwischen Industrie und Ärzten an medizinischen Hochschuleinrichtungen - unter dem Verdacht der Vorteilsannahme und Bestechlichkeit gem. $\$ \$ \$ 331,332 \mathrm{StGB}$ ?

Braun Ärztekorruption und Strafrecht - steht das ultima-ratio-Prinzip der Schaffung eines neuen Strafrechtstatbestands entgegen?, in: MedR 2013, 277-336

Dann Korruption im Notstand - Zur Rechtfertigung von Schmiergeld- und Bestechungszahlungen, in: wistra 2011, 127-132

Dannecker Die Straflosigkeit der Korruption niedergelassener Vertragsärzte als Herausforderung für den Gesetzgeber, in: ZRP 2013, 39-42

Dieners/Taschke Die Kooperation der medizinischen Industrie mit Ärzten und Krankenhäusern - Die aktuelle Rechtsprechung und ihre Konsequenzen, in: PharmR 2000, 309-321

Dölling (1996) Empfehlen sich Änderungen des Straf- und Strafprozessrechts um der Gefahr von Korruption in Staat, Wirtschaft und Gesellschaft wirksam vorzubeugen?, Gutachten C, in: Ständige Deputation des Deutschen Juristentages (Hrsg.), Verhandlungen des einundsechzigsten Deutschen Juristentages, Band I, Gutachten (Teil A bis E)

Erb (2011) Ungereimtheiten bei der Anwendung von $\$ 299$ StGB, in: Geisler u.a. (Hrsg.), Festschrift für Klaus Geppert, 97-112

Fischer (2014) Strafgesetzbuch, 61. Aufl.

Frommel Was hat der Fall Edathy mit den vom Justizministerium geplanten „Neuen Maßstäben im Kampf gegen Kinder- und Jugendpornografie“ zu tun?, in: ZRP 2014, Heft 6, im Druck

Geiger High Noon im Gesundheitswesen: Niedergelassene Vertragsärzte sind keine tauglichen Täter der Korruptionsdelikte, Besprechung des Beschlusses des Großen Senates für Strafsachen am BGH vom 29.3.2012 - GSSt 2/11, in: CCZ 2012, 172-180

Geis (Vertrags-) Arztbild des BGH versus Publikumswahrnehmung, in: GesR 2011, 641-648

Joecks/Miebach (2014) Münchner Kommentar zum StGB, 2. Aufl.

77 Scheerer KrimJ 1986, 1. Beiheft, 133,144. 
Kempf/Lüderssen/Volk (2008) Die Handlungsfreiheit des Unternehmers - wirtschaftliche Perspektiven, strafrechtliche und ethische Schranken

Kiethe Die Grenzen der strafrechtlichen Verantwortlichkeit von Bürgermeistern

- Zugleich Besprechung von BGH, Urteil v. 9. 12. 2004 - 4 StR 294/0, in: NStZ 2005, 529-534

Kindhäuser/Neumann/Paeffgen (2013) Strafgesetzbuch, 4. Aufl.

Kölbel Strafbarkeitsnahe vertragsärztliche Kooperationsformen, in: NStZ 2011, 195-200

König Neues Strafrecht gegen die Korruption, in: JR 1997, 397-404

Lackner/Kübl (2014) Strafgesetzbuch, 28. Aufl.

Larenz/Canaris (1995) Methodenlehre der Rechtswissenschaft

Laufhütte/Rissing-van Saan/Tiedemann (2012) Leipziger Kommentar zum StGB, 12. Aufl.

Obly/Sosnitza (2014) Kommentar zum Gesetz gegen den unlauteren Wettbewerb, 6. Aufl.

Pragal Das Pharma- „Marketing” um die niedergelassenen Kassenärzte: „Beauftragtenbestechung” gemäß \299 StGB!, in: NStZ 2005, 133-136

Pragal \$ 299 StGB - keine Straftat gegen den Wettbewerb!, in: ZIS 2006, 63-81

Rönnau/Golombek Die Aufnahme des „Geschäftsherrenmodells” in den Tatbestand des $\$ 299$ - ein Systembruch im deutschen StGB, in: ZRP 2007, 193-195

Säcker/Rixecker (2012) Münchner Kommentar zum BGB, 6. Aufl.

Saliger Das Untreuestrafrecht auf dem Prüfstand der Verfassung, in: NJW 2010, 3195-3198

Saliger Rechtsprobleme des Untreuetatbestandes, in: JA 2007, 326-334

Scheerer Atypische Moralunternehmer, in: KrimJ 1986, 1. Beiheft, 133-156

Schmitt-Leonardy (2013) Unternehmenskriminalität ohne Strafrecht?

Schneider/Ebermann Das Strafrecht im Dienste gesundheitsökonomischer Steuerungsinteressen - Zuweisung gegen Entgelt als „Korruption“ de lege lata und de lege ferenda, in: HRRS 2013, 219-224

Schneider (2008) Unberechenbares Strafrecht - Vermeidbare Bestimmtheitsdefizite im Tatbestand der Vorteilsannahme und ihre Auswirkungen auf die Praxis des Gesundheitswesens, in: Schneider u. a. (Hrsg.), Festschrift für Manfred Seebode, 331-350

Schneider Anmerkung zu OLG Braunschweig, Beschl. v. 23.02.2010 - Ws 17/10, in: StV 2010, 365-368 
Schneider Strafrechtliche Grenzen des Pharmamarketings, Zur Strafbarkeit der Annahme umsatzbezogener materieller Zuwendungen durch niedergelassene Vertragsärzte, in: HRRS 2010, 241-247

Schneider Wachstumsbremse Wirtschaftsstrafrecht. Problematische Folgen überzogener Steuerungsansprüche und mangelnder Randschärfe in der wirtschaftsstrafrechtlichen Begriffsbildung, in: NK 2012, 30-37

Schönke/Schröder (2014), Strafgesetzbuch, 29. Aufl.

Scbütz/ Luckmann (1979) Strukturen der Lebenswelt

Steinhilper Anmerkung zum Beschluss des OLG Braunschweig vom 23.03.2010 - Ws 17/10, in: MedR 2010, 497-502

Vogel (2004) Wirtschaftskorruption und Strafrecht - Ein Beitrag zu Regelungsmodellen im Wirtschaftsstrafrecht, in: Heinrich u.a. (Hrsg.), Festschrift für Ulrich Weber, 395-411

Wienke/Janke/Kramer Einbecker Empfehlungen der DGMR zu unerwarteten und unerwünschten Strafbarkeitsrisiken in der vertragsärztlichen Berufsausübung, in: MedR 2011, 81-86

Kontakt:

Julia Kneisel

Stud. Mitarbeiterin

Lebrstubl für Strafrecht, Strafprozessrecht, Kriminologie, Jugendstrafrecht und Strafvollzugsrecht von Herrn Prof. Dr. Hendrik Schneider

Juristenfakultät Leipzig

Burgstraße 27

04109 Leipzig

julia.kneisel@gmail.com 